\title{
Stop Stroke: A Brief Report on Door-to-Needle Times and Performance After Implementing an Acute Care Coordination Medical Application and Implications to Emergency Medical Services
}

\author{
Robert Dickson, MD, FAAEM, FACEM, FACEP; ${ }^{1}$ Adrian Nedelcut, MD ${ }^{2}$ Melissa McPeek \\ Nedelcut, $\mathrm{MD}^{2}$
}

\section{Baylor College of Medicine, Houston, Texas USA \\ 2. University of Texas, Tyler, Texas USA \\ Correspondence: \\ Robert Dickson, MD, FAAEM, FACEM, FACEP \\ Baylor College of Medicine \\ 1504 Taub Loop \\ Houston, Texas USA 77030-3411 \\ E-mail: rldickso@bcm.edu}

Conflicts of interest: Robert Dickson has stock ownership of Pulsara (Bozeman, Montana USA). The other authors have no conflicts of interest to declare.

Keywords: acute care coordination; mobile technology; stroke; therapy

\section{Abbreviations:}

AIS: acute ischemic stroke

CMS: Center for Medicare and Medicaid Services

DTN: door-to-needle

ED: emergency department

EMS: Emergency Medical Services

OTT: onset-to-treatment

TPA: Tissue Plasminogen Activator

Received: April 14, 2016

Revised: July 27, 2016

Accepted: August 6, 2016

Online publication: February 21, 2017

doi:10.1017/S1049023X17000097

\section{Abstract}

Objective: The objective of this study was to evaluate the effect of the Stop Stroke (Pulsara; Bozeman, Montana USA) medical application on door-to-needle (DTN) time in patients presenting to the emergency department (ED) with an acute ischemic stroke (AIS).

Methods: This was a retrospective cohort study of the Good Shepherd Health System (Longview, Texas USA) stroke quality improvement dashboard for a 25-month period from February 2012 through February 2014. Data analysis includes all data from Center for Medicare and Medicaid Services (CMS; Baltimore, Maryland USA) reportable cases receiving Tissue Plasminogen Activator (TPA) for AIS during the study period. The primary outcome was mean DTN times before and after initiating Stop Stroke. Secondary outcome was the effect on the DTN $\leq 60$-minute benchmark.

Results: During the study period, there were 533 stroke activations (200 before Stop Stroke implementation and 333 after). A total of 68 patients meeting inclusion criteria were analyzed (34 pre-app and 34 post- app). The observed mean DTN times post-app decreased 21 minutes (77 to 56 minutes), a $28 \%$ improvement $(\mathrm{P}=.001)$. Further, the patients meeting DTN $\leq 60$ minutes improved from $32 \%$ (11 of 34 ) to $82 \%$ (28 of 34 ) after the app's implementation.

Conclusions: In this cohort of patients with AIS, Stop Stroke improved mean DTN times and number of patients treated within 60 minutes of arrival. These results demonstrate the app's effect of increasing awareness of suspected AIS and improving coordination of care, evidenced by the magnitude of its effect on treatment times.

Dickson R, Nedelcut A, McPeek Nedelcut M. Stop Stroke: a brief report on doorto-needle times and performance after implementing an acute care coordination medical application and implications to Emergency Medical Services. Prehosp Disaster Med. 2017;32(3):343-347.

\section{Introduction and Objectives}

Acute ischemic stroke (AIS) is a major cause of morbidity and mortality with 795,000 strokes per year in the US, causing one in 20 deaths. On average in the US, a stroke occurs every 40 seconds and a death related to stroke every four minutes. ${ }^{1}$ To date, Tissue Plasminogen Activator (TPA; Activase; Genentech; San Francisco, California USA) is the only Food and Drug Administration (Silver Spring, Maryland USA) approved treatment demonstrating improved clinical outcomes for AIS. ${ }^{2-5}$ It is clear from the existing literature that time is tissue and outcomes for AIS patients receiving thrombolytic therapy are optimized when door-to-needle (DTN) times are less than 60 minutes. ${ }^{6,7}$ Emergency Medical Services (EMS) are involved in the care of these patients about two-thirds of the time. In a study of 58,000 stroke patients, improved onset-to-treatment (OTT) times were almost six times more likely with EMS involvement in their care. ${ }^{8}$ This same study demonstrated improved outcomes and less hemorrhage with every 15 minute improvement in OTT. ${ }^{8}$ There are data to suggest that pre-arrival notification by EMS improves stroke 
therapy times; however, this notification appears inconsistent and did not occur in one-quarter of EMS cases reported in a large study of over 371,000 patients. ${ }^{9,10}$ Given these associations of improved outcomes with the improved OTT times observed, EMS plays a large role in the care of these patients. At present, there are inconsistent methods used by hospital systems to coordinate this complex care.

In this study, the objective was to analyze the effect of the Pulsara Stop Stroke (Bozeman, Montana USA) medical application on DTN times in patients presenting with an AIS to a Level II emergency department (ED). The study site is part of a community medical center with an annual volume of 90,000 ED visits. The EMS service involved in care of the study patients is a rural, non-fire-based, Advanced Life Support service with 250 employees and has an annual run volume of 40,000 runs. Stop Stroke is a novel medical application developed by physicians to enhance the coordination and communication tasks essential to the rapid assessment and care of the patients suffering from an AIS. Dr. James Woodson and colleagues at Leading Edge Medical Associates, Good Shepherd Health System in Longview, Texas (USA) developed the app in 2012. The app is available free to EMS systems and commercially available to health systems who have contractual agreements with Pulsara.

Medical personnel activate the application when a stroke is identified (Figure 1). The EMS personnel, the ED, or a rapid response team can activate the app for inpatients suspected of AIS. An image is taken of the patient's clinical appearance and, along with the patient details, is transmitted to all members of the stroke care team (EMS, ED, radiologist, or neurologist on call; Figure 2). When the app is activated, these members are immediately alerted by a siren tone on the application notifying them of a new case. All members of the care team have instant access to the relevant patient information and updates in real-time (Figure 3). Pulsara apps do not store protected health information in long-term storage of mobile devices and are fully Health Insurance Portability and Accountability Act compliant.

When each link in the care team is in position to receive the patient, their readiness status is updated on the application to facilitate coordination of care (Figure 4). No specialized hardware is required, as the app is compatible with iOS and android devices. Stop Stroke provides immediate feedback on elapsed time by utilizing a universal clock comparing the current case against national benchmarks.

\section{Methods}

Data were abstracted by the Good Shepherd Health System stroke coordinator from medical records, using a standardized abstraction form, and entered into a quality improvement database (Good Shepherd stroke portal). The study included any patient arriving by EMS or private vehicle to the ED with AIS who received thrombolytic therapy between February 1, 2012 and February 28, 2014. Patients who did not meet Center for Medicare and Medicaid Services (CMS; Baltimore, Maryland USA) criteria for reporting were excluded from analysis. The CMS reporting requirements remove complicated cases requiring interval resuscitation and other confounders that prevent them from being reported to CMS for benchmarking purposes.

The stroke coordinator used a standard abstraction form and was blinded to the study objectives. The study includes all available data from the stroke dashboard, 13 months before the initiation of Stop Stroke (February 1, 2012-March 4, 2013) and 12 months

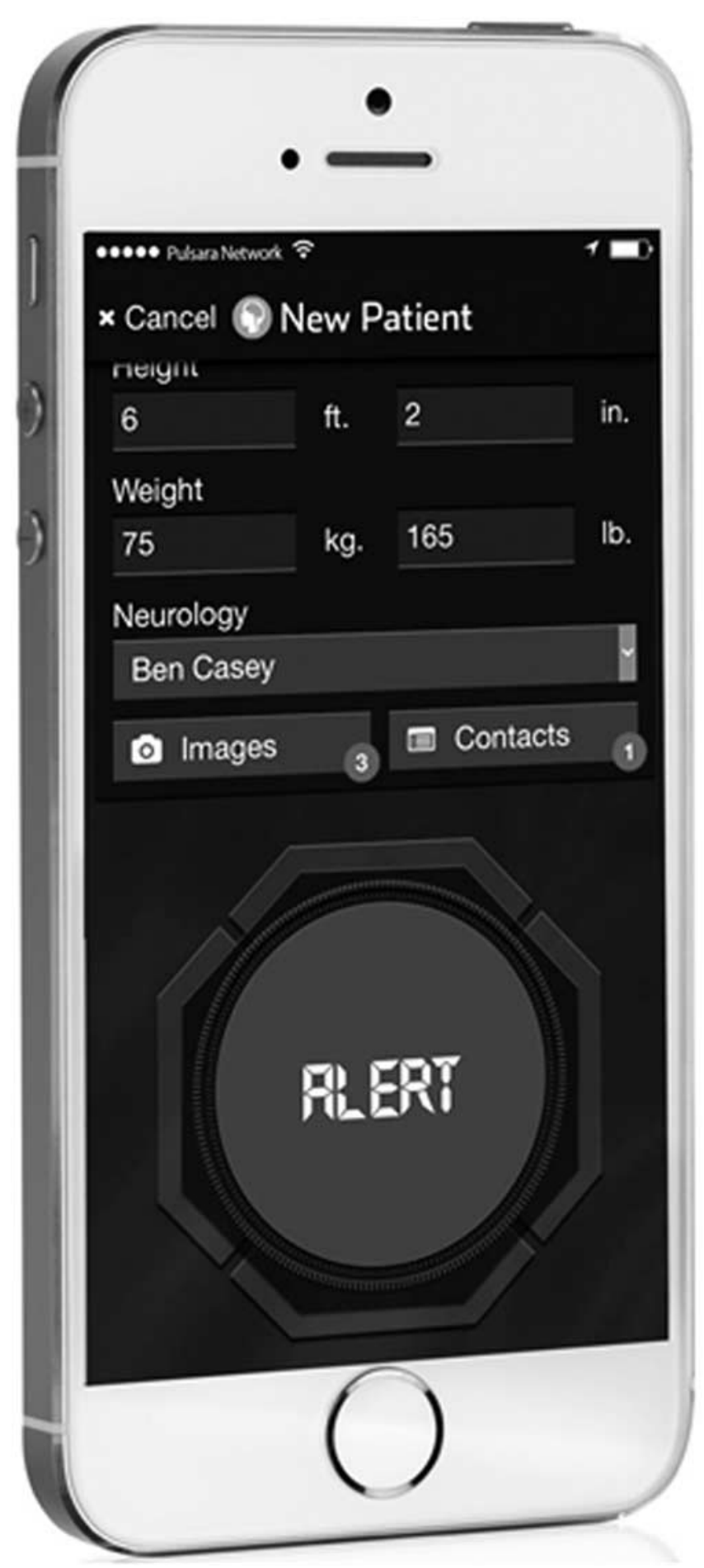

Figure 1. Pulsara Stroke Alert.

Dickson ( 2017 Prehospital and Disaster Medicine

after the application was introduced (March 5, 2013-February 28, 2014). Investigators used the recorded door and thrombolytic administration times on the stroke dashboard. Data were analyzed using Excel (Microsoft; Redmond, Washington USA) software statistics package (paired 2-tailed student's $t$ test). This study received Institutional Review Board approval from the University of Texas Health Science Center at Tyler (Texas USA). 


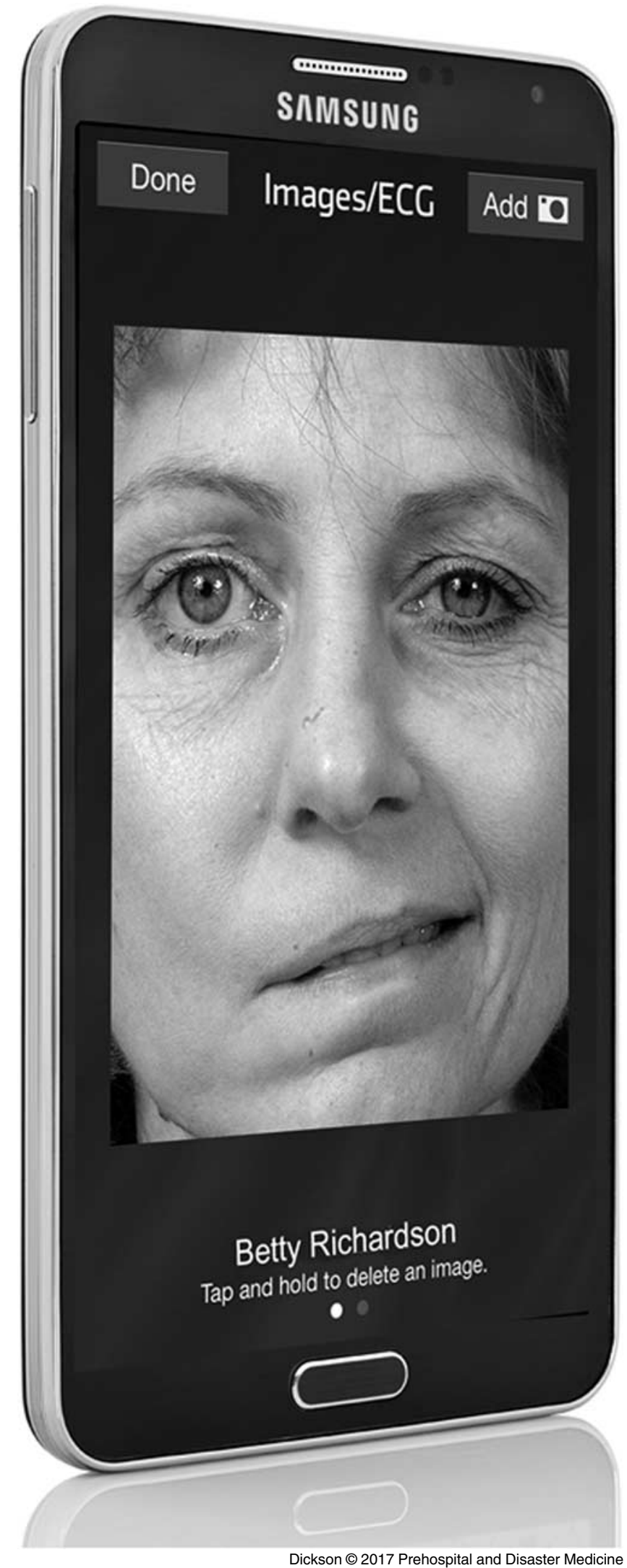

Figure 2. Pulsara Stroke Patient Image.

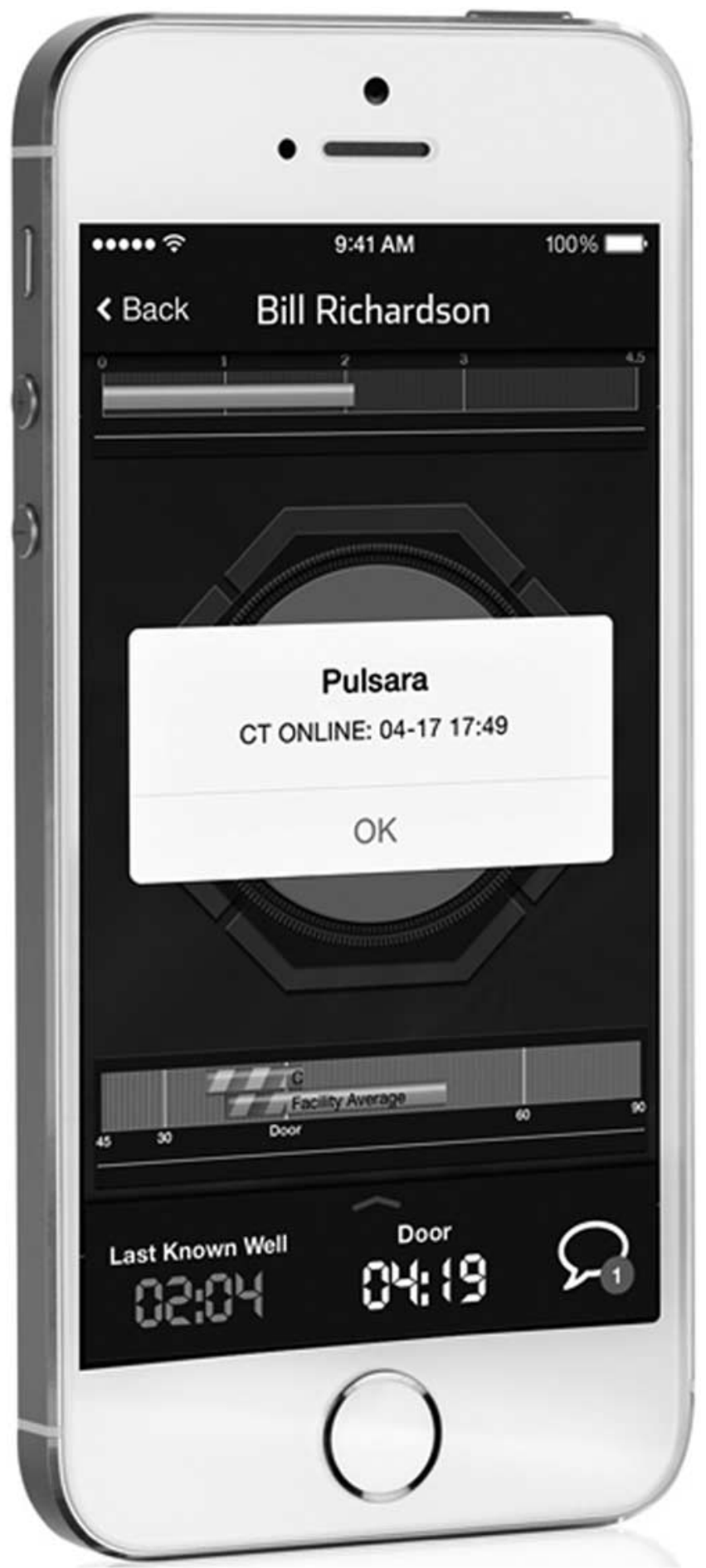

Figure 3. Pulsara Stroke Universal Clock with Notification.

\section{Results}

During the study period, 533 cases arrived with suspicion of AIS and 85 received thrombolytics (41 pre-app and 44 post-app). Seventeen cases did not meet CMS reporting criteria and were not included in the analysis (seven pre-app and 10 post-app), leaving 34 cases for analysis in both groups. The mean DTN in the pre-app group was of 77 minutes versus 56 minutes in the post-app group, a difference of 21 minutes (28\% improvement; $\mathrm{P} \leq .001 ; 95 \% \mathrm{CI}, 1-10$ minutes). 


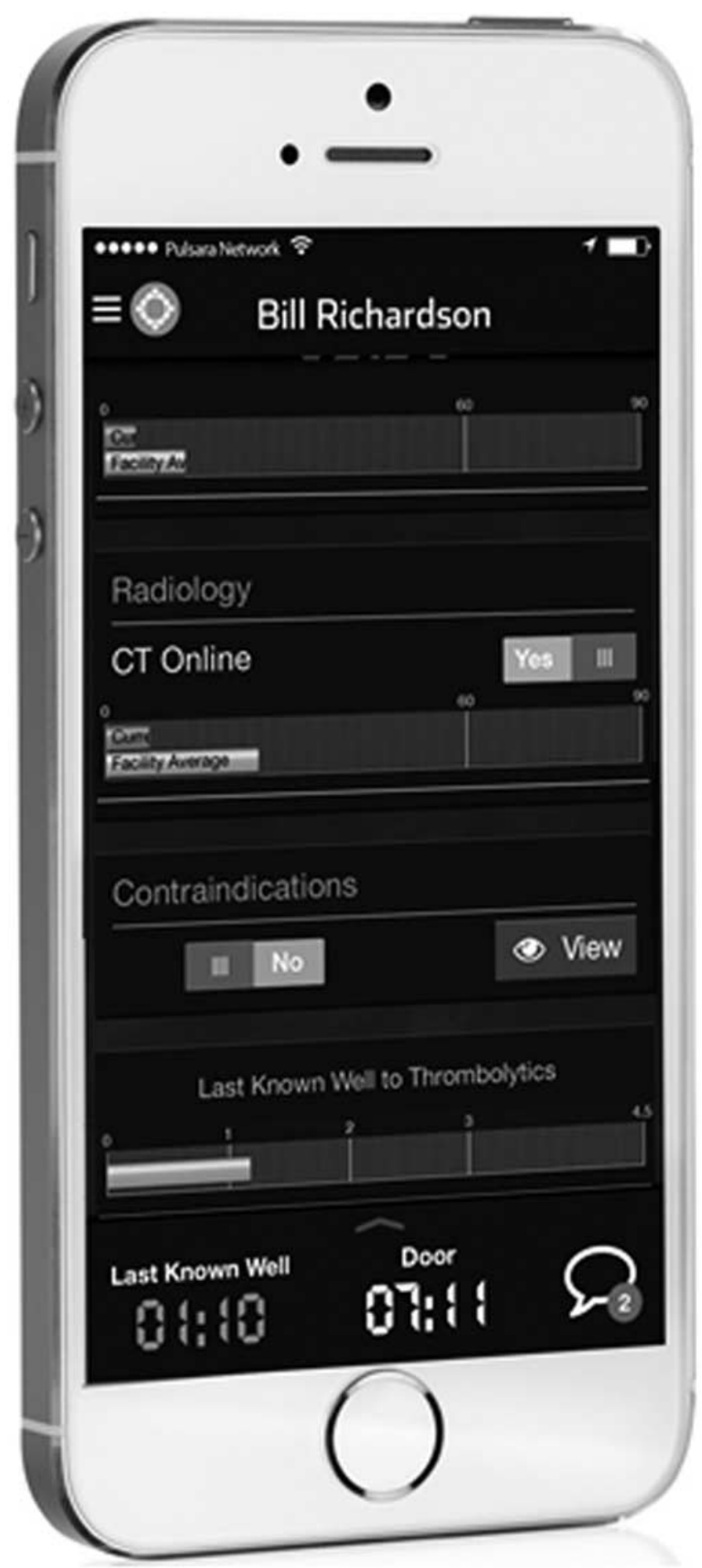

Dickson @ 2017 Prehospital and Disaster Medicine

Figure 4. Pulsara Stroke Universal Clock.

The number of cases meeting the benchmark of DTN $<60$ minutes improved from $32 \%(11 / 34)$ to $82 \%(28 / 34)$, a $155 \%(\mathrm{P}=.0001)$ relative improvement after Stop Stroke. The EMS was involved in $71 \%(24 / 34)$ of cases and there was pre-arrival notification in $66 \%$ $(16 / 24)$ of cases.

\section{Discussion}

There is mounting evidence that therapy for AIS has a far more robust effect if given in a timely manner. A study by Fonarow with over 129,000 stroke patients (of which over 25,000 received thrombolytic therapy) demonstrated that the DTN $<60$-minute group had lower in-hospital mortality and decreased symptomatic intracranial hemorrhage rates $(10.4 \%$ to $8.6 \%$ and $5.6 \%$ to $4.7 \%$, respectively). The study concludes that for every 15-minute reduction in DTN time, there was an associated improved mortality benefit of $5.0 \%{ }^{6}$

Another large study published in JAMA (American Medical Association; Chicago, Illinois USA) in 2014 demonstrated that implementing care coordination processes improved care in AIS. ${ }^{7}$ In 71,000 patients treated with thrombolytic therapy over a 10 -year period, the study demonstrated that the implementation of quality improvement measures to reduce DTN times was associated with improved rates of patients discharged home versus nursing care, $37.6 \%$ before intervention versus $42.7 \%$ after. $^{7}$

Recent studies demonstrate improved patient outcomes in AIS by augmenting current thrombolytic therapy with endovascular interventions. Their outcomes have shown reduced mortality and improved functional outcomes. ${ }^{11-13}$ The SWIFT PRIME study of combined thrombolytic/endovascular therapy demonstrated a number-needed-to-treat for improved disability outcome of 2.5 and a similar number-needed-to-treat of four for independence at long-term follow up. ${ }^{13}$

These studies achieved robust door-to-treatment times for both the TPA-only groups and the TPA + endovascular therapy groups, with median times of 55 minutes and 107 minutes, respectively. ${ }^{13}$ There are ample data to suggest that these times are not a realistic goal for most institutions, as demonstrated in a large review of over 27,000 stroke patients treated with thrombolytic therapy demonstrating only $29 \%$ were treated in the door to therapy $<60$-minute goal. ${ }^{6} \mathrm{~A}$ robust system of acute care coordination must be in place for these programs to be successful. These therapies and strategies mirror the work for improving ST Elevation Myocardial Infarction (STEMI) therapy benchmarks. A race for optimizing time from door-to-treatment (be it only thrombolytic or thrombolytic and endovascular therapy) is already underway and hospitals will be monitored and rewarded based on performance. The Stop Stroke acute care coordination app may assist in reaching these goals, as demonstrated by the improved times seen in this study.

Stop Stroke is a multi-platform electronic application that enhances coordination and communication among providers (be it inside or outside the hospital). In this study, the application had a significant impact on DTN times for patients suffering an acute stroke. Stop Stroke significantly improved an already successful operational model. The study institutions' previous DTN $<60$ minutes performance was similar to times demonstrated in a large study after quality initiative was instituted, $32 \%$ versus $29 \%$, respectively. ${ }^{6}$ It is quite possible that the positive effect of Stop Stroke may have greater impact in systems with less developed stroke care processes.

\section{Limitations}

There are some limitations to the findings reported in this investigation. A retrospective design was utilized, which has inherent biases. The center already had a robust stroke care coordination process when the app was initiated and the results may not be generalizable to other institutions. This was a single hospital study and the total numbers in both pre- and post-app groups are small; however, the data are compelling given the low cost and safety of 
this intervention. Prospective multi-center investigations are needed to fully evaluate the impact of this medical application on DTN times and patient outcomes for stroke.

\section{Conclusions}

In this cohort of patients, Stop Stroke decreased average DTN times by $28 \%$ in the patients with AIS that arrived at the

\section{References}

1. Mozaffarian D, Benjamin EJ, Go AS, et al. Heart disease and stroke statistics - 2015 update: a report from the American Heart Association. Circulation. 2015;131(4):e29-e322.

2. The National Institute of Neurological Disorders and Stroke rt-PA Stroke Study Group. Tissue plasminogen activator for acute ischemic stroke. $N$ Engl J Med. 1995;333(24):1581-1587.

3. Hacke W, Kaste M, Fieschi C, et al. Intravenous thrombolysis with recombinant tissue plasminogen activator for acute hemispheric stroke. The European Cooperative Acute Stroke Study (ECASS). JAMA. 1995;274(13):1017-1025.

4. Hacke W, Kaste M, Fieschi C, et al. Randomized double-blind placebo-controlled trial of thrombolytic therapy with intravenous alteplase in acute ischemic stroke (ECASS II). Second European-Australasian Acute Stroke Study Investigators. Lancet. 1998;352(9136):1245-1251.

5. Hacke W, Donnan G, Fieschi C, et al. Association of outcome with early stroke treatment: pooled analysis of ATLANTIS, ECASS, and NINDS rt-PA stroke trials. Lancet. 2004;363(9411):768-774.

6. Fonarow GC, Smith EE, Saver JL, et al. Timeliness of tissue-type plasminogen activator therapy in acute ischemic stroke: Patient characteristics, hospital factors, and outcomes associated with door-to-needle times within 60 minutes. Circulation. 2011;123(7):750-758
ED requiring thrombolytic therapy. Significant improvements (an increase of 155\%) in cases meeting the less than 60-minute benchmark from stroke activation to treatment also were observed. Utilization of Stop Stroke medical application by an acute stroke care program optimized the process, as demonstrated by improved time-to-treatment and percentage of cases treated in less than 60 minutes.

7. Fonarow GC, Zhao X, Smith EE, et al. Door-to-needle times for tissue plasminogen activator administration and clinical outcomes in acute ischemic stroke before and after a quality improvement initiative. JAMA. 2014;311(16):1632-1640.

8. Saver JL, Fonarow GC, Smith EE, et al. Time to treatment with intravenous Tissue Plasminogen Activator and outcome from acute ischemic stroke. JAMA. 2013; 309(23):2480-2488

9. Lin CB, Peterson ED, Smith EE, et al. Emergency Medical Service hospital prenotification is associated with improved evaluation and treatment of acute ischemic stroke. Circ Cardiovasc Qual Outcomes. 2012;5(4):514-522.

10. de la Ossa NP, Sánchez-Ojanguren J, Palomeras E, et al. Influence of the stroke code activation source on the outcome of acute ischemic stroke patients. Neurology. 2008; 70(15):1238-1243.

11. Goyal M, Demchuk AM, Menon BK, et al. Randomized assessment of rapid endovascular treatment of ischemic stroke. N Engl J Med. 2015;372(11): 1019-1030.

12. Campbell BC, Mitchell PJ, Kleinig TJ, et al. Endovascular therapy for ischemic stroke with perfusion-imaging selection. N Engl J Med. 2015;372(11):1009-1018.

13. Savor JL, Goyal M, Bonafe A, et al. Stent-retriever thrombectomy after intravenous t-PA vs. t-PA alone in stroke. $N$ Engl J Med. 2015;372(24):2285-2295 